UCRL-JC-125415

PREPRINT

\title{
A Collisional-Radiative Average Atom Model for Hot Plasmas
}

\author{
Balazs F. Rozsnyai
}

This paper was prepared for submittal to the 7th International Workshop on Radiative Properties of Hot Dense Matter

Santa Barbara, CA

November 4-8, 1996

October 17, 1996

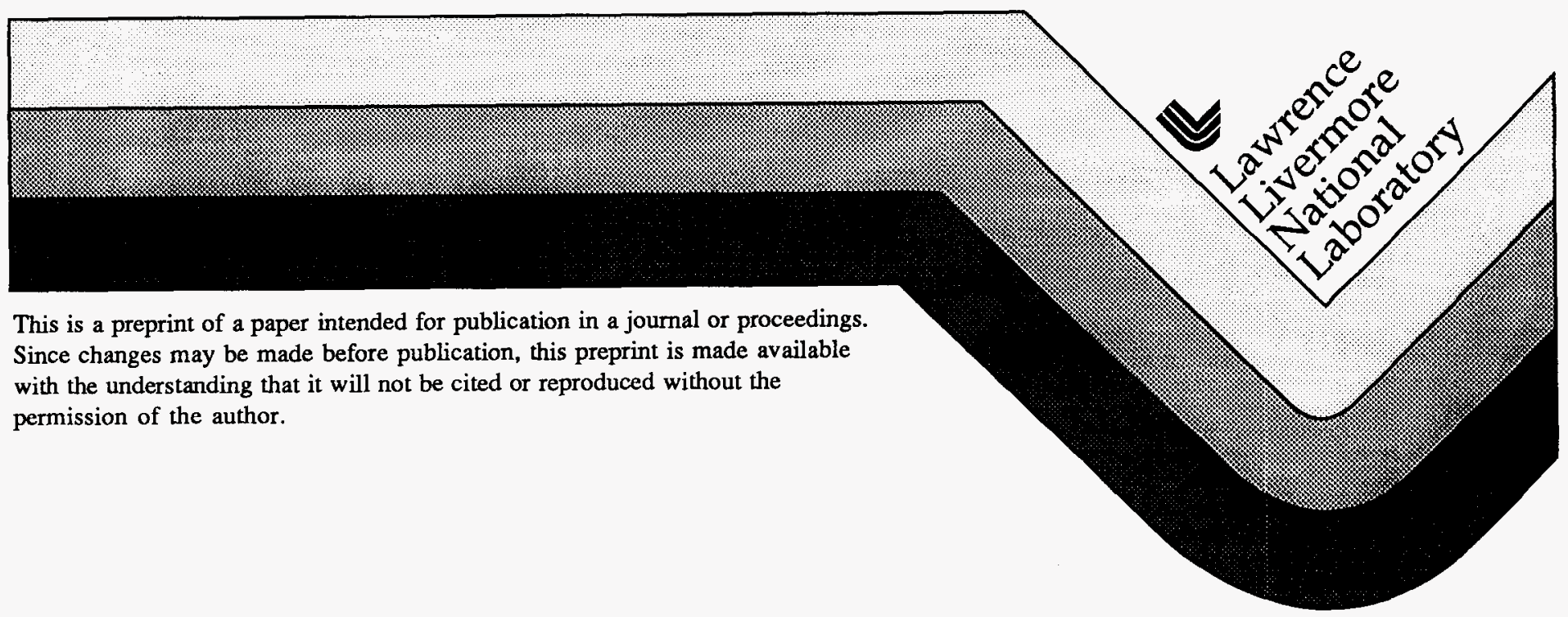




\section{DISCLAIMER}

This document was prepared as an account of work sponsored by an agency of the United States Government. Neither the United States Government nor the University of California nor any of their employees, makes any warranty, express or implied, or assumes any legal liability or responsibility for the accuracy, completeness, or usefulness of any information, apparat us, product, or process disdosed, or represents that its use would not infring eprivately owned rights. Reference herein to any specific commercial products, process, or service by trade name, trademark, manufacturer, or otherwise, does not necessarily constitute or imply its endorsement, recommendation, or favoring by the United States Government or the University of California. The views and opinions of authors expressed herein do not necessarily state or reflect those of the United States Government or the University of California, and shall not be used for advertising or product endorsement purposes. 
Prepared for the 7th International Workshop on

Radiative Properties of Hot Dense Matter

November 4-8, 1996

Santa Barbara, California 
A Collisional-Radiative Average Atom Model for Hot Plasmas.

\author{
Balazs F. Rozsnyai \\ Lawrence Livermore National Laboratory \\ P.O. Box 808 \\ Livermore, California 94550.
}

\begin{abstract}
.
A collisional-radiative "average atom" (AA) model is presented for the calculation of opacities of hot plasmas not in the condition of local thermodynamic equilibrium (LTE). The electron impact and radiative rate constants are calculated using the dipole oscillator strengths of the average atom. A key element of the model is the photon escape probability which at present is calculated for a semi infinite slab. The Fermi statistics renders the rate equations for the AA level occupancies nonlinear, which requires iterations until the steady state $\mathrm{AA}$ level occupancies are found. Detailed electronic configurations are built into the model after the self-consistent non-LTE AA state is found. The model shows a continuous transition from the non-LTE to the LTE state depending on the optical thickness of the plasma.
\end{abstract}

\title{
I. Introduction.
}

Models for calculating equation of state (EOS) data and photoabsorption cross sections of hot plasmas in the state of local thermodynamic equilibrium (LTE) have been around for some time ${ }^{1-9}$ Under LTE conditions the Fermi or Boltzmann statistics defines the distribution of the quantum mechanical states of the radiating ions thus greatly facilitating the theoretical development of the model. The condition of LTE is assured when the plasma is completely dominated by collisions and/or when the radiation field surrounding the plasma is Planckian. In the absence of the above the statistical distribution of the different ionic states can be obtained only by solving the relevant rate equations involving the ions and photons. Previous papers addressing the subject of ionization balance in terms of the steady state solutions of the rate equations used either semi-classical and parametrized atomic data, ${ }^{10}$ or hydrogenic approximation or isolated atomic data for the rate constants. ${ }^{11-15}$ The problem of obtaining a set of rate equations for medium or high- $Z$ elements in dense plasmas is complicated by a number of factors; First, the number of quantum states of the different many-electron configurations can be enormous. Second, even when data of these quantum states are available for isolated atoms and ions, the plasma electrons may sufficiently perturb those states to the degree that they may be useless. Third, the non-LTE photon distribution has to be coupled self-consistently to the statistical distribution of the many-electron ionic states. To overcome the first and second difficulty we propose a set of rate equations using the AA approach, which treats the plasma by one representative "average atom" which subsequently can be augmented with the details of the physically significant many-electron configurations. We will address the third problem with some limitations, as it will be clear later. The AA model under LTE conditions is described in Refs. 4-6 and in references given there. The AA approach to the rate equations is complicated by the fact that the Fermi statistics renders the rate equations nonlinear in terms of the AA level populations. For this reason at present we restrict our considerations to the steady-state solution of the rate equations and will not consider time-dependent problems. For the present we restrict ourselves to the case when the photon distribution is derived from a Planckian field where the energy dependent photon density is reduced by the probability of photon escape. This photon escape probability is coupled self-consistently to the quantum states of the AA atom. In Section II we present the basics of the model and in Section III we present computational results. 


\section{Description Of The Model.}

\section{A. Rate constants.}

In this sub-section we consider the rate constants which enter the rate equations. We consider excitations, deexcitations, ionizations by electron and photon impact. We assume that the electron thermalization time is short enough to assume a Maxwellian (or Fermi-Dirac) distribution for the free electrons, thus an electron temperature for the free states is well defined. Also we restrict ourselves to dipole-allowed transitions. The cross sections which determine the rate constants are approximations of precise quantum-mechanical calculations and were used before to calculate electron impact widths. ${ }^{16}$

We start with the excitation cross section form a bound AA level $i$ to an other $j$ by electron impact

$$
\sigma_{\mathrm{ij}}^{\mathrm{c}}(\mathrm{x})=1.2 \pi \mathrm{a}_{0}^{2}\left(\frac{\mathrm{e}^{2}}{\mathrm{a}_{0}}\right) \frac{\mathrm{f}_{\mathrm{ij}}}{\left(\Delta \mathrm{E}_{\mathrm{ij}}\right)^{2}}[1 .-\exp (-.3 \mathrm{x})] \frac{\ln \mathrm{x}}{\mathrm{x}}
$$

where $\Delta E_{i j}$ is the excitation energy, $f_{i j}$ is the dipole oscillator strength normalized to one electron occupancy of the initial state $i$ and computed from the AA wave functions, $x=\varepsilon / \Delta E_{i j}$ with $\varepsilon$ as the energy of the incident electron and the superscript $c$ indicates "collision". Equation (II. 1) was obtained by a series of numerical fits to $\mathrm{R}$ matrix calculations for dipole allowed transitions in medium $\mathrm{Z}$ atoms and ions. The general expression for the excitation rate is given by

$$
\mathrm{R}_{\mathrm{ij}}^{\mathrm{c}}=\int_{\Delta E_{\mathrm{ij}}}^{\infty} \sigma_{\mathrm{ij}}(\varepsilon) \mathrm{v}(\varepsilon) \mathrm{n}(\varepsilon) \mathrm{d} \varepsilon \quad\left(\sec ^{-1}\right)
$$

where $v$ stands for the velocity. In the case of Maxwellian distribution the number of free electrons between $\varepsilon$ and $\varepsilon+\mathrm{d} \varepsilon$ is given by

$$
\begin{aligned}
\mathrm{n}(\varepsilon) \mathrm{d} \varepsilon= & \frac{2}{\sqrt{\pi}} \rho \frac{1}{(\mathrm{kT})^{3 / 2}} \varepsilon^{1 / 2} \exp (-\varepsilon / \mathrm{kT}) \mathrm{d} \varepsilon= \\
& \frac{2}{\sqrt{\pi}} \rho \frac{1}{(\mathrm{kT})^{3 / 2}}\left(\Delta \mathrm{E}_{\mathrm{ij}}\right)^{3 / 2} \mathrm{x}^{1 / 2} \exp \left(-\mathrm{x} \Delta \mathrm{E}_{\mathrm{ij}} / \mathrm{kT}\right) \mathrm{dx}
\end{aligned}
$$

where $k T$ stands for the temperature (in energy units) of the free electron gas with $k$ denoting Boltzmann's constant and $\rho$ for the total free electron density. Using $v=c\left(2 \varepsilon / \mathrm{mc}^{2}\right)^{1 / 2}$ we obtain

$$
\begin{aligned}
R_{i j}^{c}=1.2\left(\frac{8 \pi}{m c^{2}}\right)^{1 / 2} \frac{f_{i j}}{(k T)^{3 / 2}} e^{+} c \rho \int_{1}^{\infty}[1 .-\exp (-.3 x)] \ln x \exp \left(-x \Delta E_{i j} / k T\right) d x= \\
=f_{i j} j_{i j}^{e x}\left(\sec ^{-1}\right)
\end{aligned}
$$

where we factored out the dipole oscillator strength. The integral in Eq.(4) is given by 


$$
\frac{k T}{\Delta E_{i j}} E_{1}\left(\frac{\Delta E_{i j}}{k T}\right)-\frac{k T}{\Delta E_{i j}+.3 k T} E_{1}\left(\frac{\Delta E_{i j}+.3 k T}{k T}\right)
$$

where $E_{1}$ stands for the exponential integral. For the de-excitation (superelestic) rates we have

$$
R_{j i}^{c}=\frac{f_{j i}}{f_{i j}} \exp \left(\Delta E_{i j} / k T\right) R_{i j}^{c} \rightarrow \text { detailed balance }
$$

The last relation is due to the fact that the de-excitation cross section is the same as (II. 1) except the $\ln (x)$ term has to be replaced by $\ln (x+1)$.

We calculate the electron impact ionization rates fro the cross section

$$
\sigma_{i c}^{c}(x)=\frac{K}{\varepsilon_{i}^{2}} e^{4}[1-3 \exp (x)] \frac{\ln x}{x} ; x=\frac{\varepsilon}{\left|\varepsilon_{i}\right|}
$$

where $\varepsilon$ and $\varepsilon_{\mathrm{i}}$ stand for the energy of the incident electron and for the eigenenergy of the bound level L, respectively and the subscript $c$ indicates the transition to a continuum state. Equation (II. 7) is analogous to Eq.(II. 1) and apart from the exponential term to Lotz's cross section for electron impact ionization ${ }^{17}$ For the best fit to agree with more sophisticated calculations we use $K=2.27$. The ionization rate constant is given by

$$
\mathrm{R}_{\mathrm{ic}}^{\mathrm{c}}=\mathrm{Ke}^{4} \frac{2^{3 / 2}}{\left.(\pi \mathrm{mc})^{2}\right)^{1 / 2}} \frac{\mathrm{c} \rho}{(\mathrm{kT})^{3 / 2}} \int_{1}^{\infty}[1 .-\exp (-.3 \mathrm{x})] \ln \mathrm{x} \exp \left(-\mathrm{x} \mid \varepsilon_{\mathrm{i}} / / \mathrm{kT}\right) \mathrm{dx} \quad\left(\mathrm{sec}^{-1}\right)
$$

The condition of detailed balance relates the three-body recombination to Eq.(II. 8) by

$$
\mathrm{R}_{\mathrm{ci}}^{\mathrm{c}}=\exp \left[\left(\mu-\varepsilon_{\mathrm{i}}\right) / \mathrm{kT}\right] \mathrm{R}_{\mathrm{ic}}^{\mathrm{c}}
$$

where $\mu$ is the Fermi level of the free electron gas. Since $\exp (\mu / \mathrm{kT})$ is proportional to $\rho$ and so is $R_{i c}^{c}$, Eq.(II. 10) indeed represents three-body recombination. The condition $N_{i} R_{i c}^{c}=\left(g_{i}-N_{i}\right) R_{c i}^{c}$ leads to the Fermi statistics where $N_{i}$ stands for the population of level $i$ and $g_{i}$ for its statistical weight.

We account for the dielectronic recombination and its inverse by the Auger matrix elements $A_{j i}^{m c}=$ Auger process, an electron from level $m$ goes to continuum while an other from level $j$ fills a hole state in $\mathrm{I}$. In the AA approximation this matrix element is given by ${ }^{18}$

$$
A_{j i}^{m c}=\frac{2 \pi}{\hbar} N_{j} N_{m} \sum_{l_{c}}\left(2 l_{i}+1\right)\left(2 l_{c}+1\right) a_{i} a_{c}\left(B_{1}+B_{2}+B_{12}\right)
$$

where

$$
B_{1}=\sum_{k} \frac{1}{2 k+1}\left(\begin{array}{ccc}
1_{j} & k & 1_{i} \\
0 & 0 & 0
\end{array}\right)^{2}\left(\begin{array}{ccc}
l_{m} & k & l_{c} \\
0 & 0 & 0
\end{array}\right)^{2}\left[R^{k}\left(n_{j} l_{j} n_{m} l_{m} ; n_{i} l_{i} c l_{c}\right]^{2}\right.
$$




$$
\begin{aligned}
B_{2} & =\sum_{p} \frac{1}{2 p+1}\left(\begin{array}{ccc}
l_{m} & p & l_{i} \\
0 & 0 & 0
\end{array}\right)^{2}\left(\begin{array}{ccc}
1_{j} & p & l_{c} \\
0 & 0 & 0
\end{array}\right)^{2}\left[R^{p}\left(n_{m} l_{m} n_{j} l_{j} ; n_{i} l_{i} c_{c}\right]^{2}\right. \\
B_{12} & =(-1)^{\lambda} \sum_{k} \frac{1}{2 k+1}\left(\begin{array}{ccc}
l_{j} & k & 1_{i} \\
0 & 0 & 0
\end{array}\right)\left(\begin{array}{ccc}
l_{m} & k & 1_{c} \\
0 & 0 & 0
\end{array}\right) \sum_{p} \frac{1}{2 p+1}\left(\begin{array}{ccc}
l_{m} & p & 1_{i} \\
0 & 0 & 0
\end{array}\right)\left(\begin{array}{ccc}
l_{j} & p & 1_{c} \\
0 & 0 & 0
\end{array}\right) x \\
\left\{\begin{array}{lll}
l_{m} & l_{c} & k \\
l_{j} & l_{i} & p
\end{array}\right\} R^{k}\left(n_{j} l_{j} n_{m} l_{m} ; n_{i} l_{i} l_{c}\right) R^{p}\left(n_{m} l_{m} n_{j} l_{j} ; n_{i} l_{i} c l_{c}\right) & \text { (II. 10c) }
\end{aligned}
$$

where $\lambda=1_{j}+l_{i}+l_{m}+l_{c}+1$ and the R-s stand for the usual Slater integrals. Also, in Eq.(II. 10a) the a-s stand for the availability of the states $i$ and $c$. The symbols $n$ and $l$ stand for the principal and angular momentum quantum numbers, respectively. It should be noted that Eqs.(II. 10a)-(II. 10d) have nothing to do with the thermal state of the plasma. They are strictly the results of taking the Auger matrix elements between averaged $\mathrm{J}$ states.

In the case of photoabsorption we consider the photon escape probability due to the finite optical thickness. The concept of photon escape probability was used in previous work in connection with line transfers. ${ }^{19-21}$ In this work we calculate the photon escape probability form the total self-consistent photoabsorption cross section, which includes the line profiles together with photoinization and inverse bremsstrahlung. We assume that the radiating ion is situated in a semi infinite slab at a distance $d$ away from the edge of the slab. For this case it is easy to show that the photon escape probability is given by

$$
P(\hbar \omega)=\exp (-x)-x E_{1}(\dot{x})
$$

where $x=d / 1 ; 1=\frac{1}{\sigma(\hbar \omega) D}$

with $\sigma$ and $D$ as the photoabsorption cross section and ion density of the plasma. Actually, we will use the phote $n$ confinement probability which is $1-P_{c}$.

Next, we consider the radiative transitions. For a spontaneous downward transition we have Einstein's transition probability

$$
A_{j i}=\frac{2 \alpha}{\hbar} \frac{\left(\Delta E_{i j}\right)^{2}}{m c^{2}} f_{j i}\left(\sec ^{-1}\right)
$$

For an induced transition we use the general form

$$
B_{\mathrm{ij}}=c \int \sigma_{\mathrm{ij}}(\hbar \omega) \mathrm{N}(\hbar \omega) \mathrm{d}(\hbar \omega)\left(\sec ^{-1}\right)
$$

where $N(\hbar \omega) \mathrm{d}(\hbar \omega)$ is the number of photons /cc in the energy interval $\hbar \omega$ and $\hbar \omega+\mathrm{d}(\hbar \omega)$.

For the absorption cross section we have 


$$
\sigma_{\mathrm{ij}}(\hbar \omega)=\frac{2 \pi^{2} \mathrm{e}^{2}}{\mathrm{mc}} \hbar \mathrm{f}_{\mathrm{ij}} \mathrm{b}_{\mathrm{ij}}(\hbar \omega) \quad\left(\mathrm{cm}^{2}\right)
$$

where $b_{\mathrm{ij}}(\hbar \omega)$ is the line shape function of the transition. Assuming a Dirac $\delta$ function for the line shape we have

$$
\mathrm{B}_{\mathrm{ij}}=\frac{2 \pi^{2} \mathrm{e}^{2}}{\mathrm{~m}} \hbar \mathrm{f}_{\mathrm{ij}} \mathrm{N}\left(\hbar \omega_{\mathrm{ij}}\right)
$$

For a Planckian radiation field we have

$$
N_{P}(\hbar \omega)=\frac{\omega^{2}}{\hbar \pi^{2} c^{3}} \frac{1}{\exp (\hbar \omega / k T)-1}
$$

We mimic the finite optical thickness with a photon distribution $N(\hbar \omega)=N_{p}(\hbar \omega) P_{c}(\hbar \omega)$ and obtain

$$
B_{i j}=\frac{2 \alpha}{\hbar} \frac{\left(\hbar \omega_{i j}\right)^{2}}{m c^{2}} f_{i j} \frac{1}{\exp \left(\hbar \omega_{i j}\right)-1} P_{c}\left(\hbar \omega_{i j}\right)
$$

For the downward $j-i$ transition the combined spontaneous and induced rates give

$$
A_{j i}+B_{j i}=\frac{2 \alpha}{\hbar} \frac{\left(\Delta E_{i j}\right)^{2}}{m c^{2}} f_{j i}\left[1+\frac{1}{\exp \left(\hbar \omega_{i j} / k t\right)-1} P_{c}\left(\hbar \omega_{i j}\right)\right]
$$

For the photoionization rate from a bound state $m$ to the continuum $c$ we have

$$
\mathrm{r}_{\mathrm{mc}}^{\mathrm{r}}=\frac{8 \pi}{\mathrm{h}^{3} \mathrm{c}^{2}} \int_{\left|\varepsilon_{\mathrm{m}}\right|}^{\infty}(\hbar \omega)^{2} \sigma_{\mathrm{m}, \mathrm{c}}(\hbar \omega) \mathrm{P}_{\mathrm{c}}(\hbar \omega) \exp (-\hbar \omega) \mathrm{d}(\hbar \omega)\left(\sec ^{-1}\right)
$$

where $P_{c}$ stands for the frequency dependent photon confinement factor and $\sigma_{m . c}$ is the photoionization cross section of the level $m$ normalized to one electron occupancy. It should be noted that Eq.(Il. 19) includes the induced downward $c \rightarrow m$ transition. The inverse radiative recombination is given by

$$
\mathrm{R}_{\mathrm{cm}}^{\mathrm{r}}=\exp \left[\left(\mu-\varepsilon_{\mathrm{m}}\right) / \mathrm{kT}\right] \mathrm{R}_{\mathrm{mc}}^{\mathrm{r}}
$$

where $R_{c m}^{r}$ refers to the photoionization rate in the optically thick case when $P_{c}=1$ and reflects the principle of detailed balance when the free electrons follow the Fermi statistics. 


\section{B. Rate equations.}

First, we summarize the rate constants which drive the rate equations;

$\mathrm{R}_{\mathrm{mk}}^{\mathrm{c}}=$ excitation (deexcitation from level $\mathrm{m}$ to level $\mathrm{k}$ by electron impact.

$\mathrm{R}_{\mathrm{mk}}^{\mathrm{r}}=$ the same for radiative transitions (spontaneous and induced combined)

$\mathrm{R}_{\mathrm{mc}}^{\mathrm{c}}=$ electron impact ionization from level $\mathrm{m}$ to continuum.

$\mathrm{R}_{\mathrm{cm}}^{\mathrm{c}}=\exp \left[\left(\mu-\varepsilon_{\mathrm{m}}\right) / \mathrm{kT}\right] \mathrm{R}_{\mathrm{m} . \mathrm{c}}^{\mathrm{c}}=$ the reverse, three body recombination

$\mathrm{r}_{\mathrm{mc}}^{\mathrm{r}}=$ photoionization

$\mathrm{R}_{\mathrm{cm}}^{\mathrm{I}}=\exp \left[\left(\mu-\varepsilon_{\mathrm{m}}\right) / \mathrm{kT}\right] \mathrm{R}_{\mathrm{m} . \mathrm{c}}^{\mathrm{r}}=$ the reverse, radiative recombination

$\mathrm{A}_{\mathrm{ji}}^{\mathrm{mc}}=$ Auger process, an electron from level $\mathrm{m}$ goes to continuum while an other from level $\mathrm{j}$ fills a hole state in $i$.

$\mathrm{A}_{\mathrm{ij}}^{\mathrm{cm}}=$ the inverse.

$N_{c}=$ the number of free electrons per AA.

All rate constants are normalized to one electron occupancies of the bound levels and they do not include the availability of the final state. Assuming that the number of bound AA levels is finite the rate equations are given by

$$
\begin{aligned}
& \frac{d N_{m}}{d t}=-\left[\sum_{k=1}^{b}\left(R_{m k}^{c} N_{c}+R_{m k}^{r}\right) a_{k}\right] N_{m}-\left(R_{m . c}^{c} N_{c}+R_{m . c}^{r}\right) N_{m} \\
& \frac{-\left(\sum_{j} \sum_{i} N_{j}\left(g_{i}-N_{i}\right) A_{j i}^{m c}\right) N_{m}+\left[\sum_{k=1}^{b}\left(R_{k m}^{c} N_{c}+R_{k m}^{r}\right) N_{k}\right] a_{m}+}{+\left(R_{c m}^{c} N_{c}+R_{c m}^{r}\right)\left(g_{m}-N_{m}\right)+} \\
& \left(\sum_{j} \sum_{i}\left(g_{j}-N_{j}\right) N_{i} A_{i j}^{c m}\right)\left(g_{m}-N_{m}\right)
\end{aligned}
$$




$$
\begin{aligned}
& \frac{\mathrm{dN}_{\mathrm{c}}}{\mathrm{dt}}= \sum_{\mathrm{m}}\left(\mathrm{R}_{\mathrm{mc}}^{\mathrm{c}} \mathrm{N}_{\mathrm{c}}+\mathrm{R}_{\mathrm{mc}}^{\mathrm{r}}\right) \mathrm{N}_{\mathrm{m}}-\sum_{\mathrm{m}}\left(\mathrm{R}_{\mathrm{cm}}^{\mathrm{c}} \mathrm{N}_{\mathrm{c}}+\mathrm{R}_{\mathrm{cm}}^{\mathrm{r}}\right)\left(\mathrm{g}_{\mathrm{m}}-\mathrm{N}_{\mathrm{m}}\right)+ \\
& \sum_{\mathrm{m}} \sum_{\mathrm{j}} \sum_{\mathrm{i}}\left[\mathrm{N}_{\mathrm{j}} \mathrm{N}_{\mathrm{m}}\left(\mathrm{g}_{\mathrm{i}}-\mathrm{N}_{\mathrm{i}}\right) \mathrm{A}_{\mathrm{ji}}^{\mathrm{mc}}-\mathrm{N}_{\mathrm{i}}\left(\mathrm{g}_{\mathrm{m}}-\mathrm{N}_{\mathrm{m}}\right)\left(\mathrm{g}_{\mathrm{j}}-\mathrm{N}_{\mathrm{j}}\right) \mathrm{A}_{\mathrm{ij}}^{\mathrm{cm}}\right]
\end{aligned}
$$

All summations go over the bound level indices. It is easy to see that Eqs.(II. 23) and (II. 24) satisfy the particle conservation

$$
\sum_{m} \frac{d N_{m}}{d t}+\frac{d N_{c}}{d t}=0 \quad \text { because }
$$

the sums with respect to $\mathrm{m}$ in 1 and 4 cancel out, the sum of 2 with respect to $\mathrm{m}$ cancels out 7 , the same for 3 and 8,5 and 9 and 6 and 10 . The above is simply the consequence of particle number conservation. In a steady state solution we must have $\frac{\mathrm{dN}_{\mathrm{c}}}{\mathrm{dt}}=0$ separately, which means that the sums 789 and 10 have to add up to zero. This last condition defines the steady state ionization balance, LTE or not. Next, we introduce some new quantities from the triple sums 9 and 10.

Let

$$
\sum_{j} \sum_{i} N_{j}\left(g_{i}-N_{i}\right) A_{j i}^{m c}=K_{m c}
$$

and

$$
\sum_{j} \sum_{i} N_{i}\left(g_{j}-N_{j}\right) A_{i j}^{c m}=\exp \left[\left(\mu-\varepsilon_{m}\right) / k T\right] K_{c m}
$$

Using the relationships (II. 21) and (II. 22) for the detailed balance and then Eq.(II. 24) for the steady state case has the general form

$$
\begin{aligned}
\frac{\mathrm{dN}_{\mathrm{c}}}{\mathrm{dt}}=\sum_{\mathrm{m}}\left\{\left(\mathrm{R}_{\mathrm{m} \cdot \mathrm{c}}^{\mathrm{c}} \mathrm{N}_{\mathrm{c}}+\mathrm{r}_{\mathrm{m} \cdot \mathrm{c}}^{\mathrm{r}}+\mathrm{K}_{\mathrm{m} \cdot \mathrm{c}}\right) \mathrm{N}_{\mathrm{m}}-\exp \left[\mu-\varepsilon_{\mathrm{m}}\right) / \mathrm{kT}\right]\left(\mathrm{g}_{\mathrm{m}}-\mathrm{N}_{\mathrm{m}}\right) \mathrm{x} \\
\left.\left(\mathrm{R}_{\mathrm{m} . \mathrm{c}}^{\mathrm{c}} \mathrm{N}_{\mathrm{c}}+\mathrm{R}_{\mathrm{m} \cdot \mathrm{c}}^{\mathrm{r}}+\mathrm{K}_{\mathrm{c} \cdot \mathrm{m}}\right)\right\}=0
\end{aligned}
$$

Equation (II. 26) is generally valid for LTE or non-LTE ionization balance. Next, we take the approximation that the individual $\mathrm{m}$ terms in Eq.(11. 269) are zero, which we will call as the "one level in continuum" (OLC) approximation. The name is justified by observing that the OLC approximation is exact when there is only one bound level embedded in the continurum. The OLC approximation yields for the occupancy of the level $m$ 


$$
N_{m}=\frac{g_{m} \lambda_{m}}{\exp \left[\left(\mu-\varepsilon_{m}\right) / k T\right]+\lambda_{m}}
$$

where

$$
\lambda_{m}=\frac{R_{m, c}^{c}+R_{m . c}^{r}+K_{c . m}}{R_{m, c}^{c}+r_{m, c}^{r}+K_{m . c}}
$$

In the optically thick case when $P_{c}=1 \lambda_{m}=1$ and Eq.(II. 27) yields the Fermi statistics. $N_{c}$, the number of free electrons per AA is obviously $Z$ - the sum of bound electrons.

The individual occupancies of the bound levels given by Eqs.(II. 27) and (II. 28) are not exact, but their sum satisties Eq. (II. 26), therefore we adopt it as a reasonable approximation for the ionization state. This facilitates the iteration procedure of solving Eq.(II. 23) by determining $N_{c}$ from Eqs.(II. 27) and (II. 28) first and solving Eq.(II. 23) for the bound state populations subsequently. In fact, the selfconsistent solutions of Eqs.(II. 23), (II. 27) and (II. 28) together with Eq.(II. 11) for the photon escape probability involve a hierarchy of iterations that we outline in the next Section.

\section{Computational Results.}

The rate equations (II. 23) and (II. 24) in terms of the bound level occupancies and $N_{c}$ are cubic, which necessitates the application of iteration schemes for the solution. In addition, all solutions must be also self-consistent with the frequency dependent photon escape probability. In order to make the whole problem computationally tractable we adopted the following iteration scheme:

1. We start from the self-consistent LTE problem as described in Refs. 6 and 8 and compute all the rate constants from the LTE wave functions and from the LTE photon escape probability.

2. Next we obtain the first iteration value for $N_{c}$ in the OLC approximation using Eqs.(II. 27) and (II. 28).

3. Next we solve Eq.(II. 23) for the bound level populations by successive iterations where the availability factors and the factors $\left(\mathrm{g}_{\mathrm{m}}-\mathrm{N}_{\mathrm{M}}\right)$ for the $\mathrm{n}$-th iteration are taken from the $\mathrm{n}-\mathrm{l}$ th iteration and we iterate until the values of $\mathrm{N}_{\mathrm{m}}$ converge. At this point the $\mathrm{AA}$ problem is solved in first iteration.

4. In order to obtain realistic photoabsorption cross sections and photon escape probabilities one must go beyond the AA model and build into the model the effect of "detailed configuration accounting" (DCA) due to the many-electron configurations. This necessitates to calculate the statistical distribution of the numerous DCA states which in a system not in LTE is a serious problem in itself. We solve this problem by calculating an "equivalent LTE temperature" $k T_{\text {eq }}$, which after Busquet ${ }^{22}$ is defined as the temperature which under LTE condition yields the same value for $\mathrm{N}_{c}$ as the nonLTE problem. We also calculate an equivalent Fermi level $\mu_{e q}$ which together with $\mathrm{kT}_{\mathrm{eq}}$ is used to calculate an equivalent Boltzmann distribution of the many-electron DCA states. We proceed to compute the photoabsorption from these DCA states as described in Ref. 8 
5. Having obtained the non-LTE photoabsorption cross section and non-LTE populations in this manner in the first iteration we go back to point 1 and iterate until convergency is reached. In the calculations presented here convergency was reached usually after 4 or 5 iterations.

All the above iterations make the calculations rather lengthy compared to the LTE problems.

We present two sets of calculations, one for praseodymium $(Z=59)$ at $\mathrm{kT}=1 \mathrm{KeV}$ and at $0.1 \mathrm{~g} / \mathrm{cc}$ density and one for germanium $(Z=32)$ at $\mathrm{kT}=0.5 \mathrm{KeV}$ and at $0.01 \mathrm{~g} / \mathrm{cc}$ density. Calculations for the first case for an optically thin plasma and using the method of "equivalent LTE temperature" were published in Ref. 18, the second was a study case at the WorkOp-III:94 conference where the author presented non-LTE corona equilibrium opacities obtained from level populations predicted by the LASNEX code of the Lawrence Livermore National Laboratory. In this report we present calculations for various optical thicknesses and we show the results converge, as expected, to the LTE case when the plasma gractually becomes optically thick. We label the parameter for characterizing the optical thickness by $\mathrm{x}_{\mathrm{R}}$ which is the distance of the central ion from the edge of the semi-infinite slab divided by the LTE Rosseland mean length $L_{R}$

The conditions are summarized in Table $I$ where we give the parameters $X_{R}$ in column 1 , the equivalent LTE temperatures in column 2, the numbers of free electrons per AA in column 3 and the degeneracy parameters of the free electron gas $\mu / \mathrm{kT}$ and $\mu_{\text {eq }} / \mathrm{kT}_{\text {eq }}$ in columns 4 and 5 , respectively.

The calculations for praseodymium are shown in Figs. 1-7, and also in Figs. 12 and 13. Figure 1 shows the self consistent photon confinement factors $P_{c}$ for three cases of $x_{R}$. Figure 2 shows the Fermi functions, defined as $\mathrm{N}_{\mathrm{m}} / \mathrm{g}_{\mathrm{m}}$ versus the binding energies (in au-s) of the self consistent AA levels for four cases of optical thickness together with the LTE case. We can see that curve IV, corresponding to the case when the radiating ion is one Rosseland mean length away from the edge of the slab, practically coincides with the LTE curve, even when the photon confinement factors are not 1 for all photon energies. Figures 3 and 4 compare the LTE Fermi functions with those calculated in the OLC approximation and with the full non-LTE rate equations.

The calculated opacities for praseodymium are shown on Figs. 5, 6 and 7 for $x_{R}=0,10^{-4}$ and $10^{-2}$, respectively. In each figure we compare the LTE opacities with that of the full non-LTE calculations and also with the LTE calculations using the equivalent temperatures of Table I. For the non-LTE calculations the distribution of the DCA sates are computed with the aid of the equivalent LTE temperatures and Fermi level. The number of DCA states which contribute significantly to the opacities can be quite numerous, for example the opacity in Fig. 6 was obtained using 144 DCA configurations distributed over 12 different degrees of ionization. It is out of the scope of this paper to discuss further details of the DCA states and such details of the opacity calculations as line profiles, bremsstrahlung and photoionization. For those the reader is referred to Refs. 4, 6, 8, and 15 and references quoted there. Here we concentrate only on the non-LTE AA level populations. The calculated opacities for the germanium case are shown in Figs. 8, 9, 10 and 11. Figures 8, 9, and 10 are analogous to those of Figs. 5,6 , and 7. In Fig. 11 we compare the non-LTE opacities with $x_{R}=10^{-4}$ and $10^{-2}$ with that of an earlier calculation where the non-LTE AA populations were obtained by the radiation transport code LASNEX. Since the LASNEX code uses a somewhat simple atomic physics package, the calculations based on LASNEX based populations are not self-consistent, thus the apparent differences are not surprising. It should be noted that the LASNEX populations were obtained for the optically thin case $\left(x_{R}=0\right)$. 
Finally, in Fig. 12 we investigate the effect of the presence or absence of the Auger matrix elements for one case of the praseodymium set. It is evident that in the absence of the Auger transitions the occupancies of the upper levels are depleted, thus reducing the photoabsorption at low photon energies, as shown in Fig. 13.

\section{Discussion.}

The purpose of this paper was to present a somewhat rudimentary model for estimating the non-LTE effects on photoabsorption in laboratory plasmas. These effects may be quite important in laser produced plasmas. Although some experimental works have been done to measure LTE opacities of hot plasmas, the author is not aware of experimental works concentrating on non-LTE effects. Neither is clear to the author at this point how to design meaningful experiments which clearly distinguishes between the LTE and non-LTE state of the plasma. Hopefully, this will come in the future.

\section{Acknowledgment}

This work was performed under the auspices of the U.S. Department of Energy by Lawrence Livermore National Laboratory under Contract No. W-7405-Eng-48. 
Finally, in Fig. 12 we investigate the effect of the presence or absence of the Auger matrix elements for one case of the praseodymium set. It is evident that in the absence of the Auger transitions the occupancies of the upper levels are depleted, thus reducing the photoabsorption at low photon energies, as shown in Fig. 13.

\section{Discussion.}

The purpose of this paper was to present a somewhat rudimentary model for estimating the non-LTE effects on photoabsorption in laboratory plasmas. These effects may be quite important in laser produced plasmas. Although some experimental works have been done to measure LTE opacities of hot plasmas, the author is not aware of experimental works concentrating on non-LTE effects. Neither is clear to the author at this point how to design meaningful experiments which clearly distinguishes between the LTE and non-LTE state of the plasma. Hopefully, this will come in the future.

\section{Acknowledgment}

This work was performed under the auspices of the U.S. Department of Energy by Lawrence Livermore National Laboratory under Contract No. W-7405-Eng-48. 
Table I. Equivalent LTE temperatures, ionization sates and Fermi levels.

1. $\operatorname{Pr}(Z=59)$ at $K T=1 \mathrm{keV}$ and $.1 \mathrm{~g} / \mathrm{cc} . \mathrm{L}_{\mathrm{R}}=1.3619 \mathrm{~cm}$.

$\begin{array}{llccc}\mathrm{X}_{R} & \mathrm{kT} \mathrm{T}_{\text {eq }}(\mathrm{KeV}) & \mathrm{N}_{\mathrm{c}} & -\mu / \mathrm{kT} & -\mu_{\text {eq }} / \mathrm{kT} \text { eq } \\ & & & & \\ 0.0000 & 0.20313 & 28.81 & 9.6756 & 7.3283 \\ 0.0001 & 0.44531 & 40.93 & 9.3293 & 8.1022 \\ 0.01 & 0.84375 & 49.01 & 9.1515 & 8.9023 \\ 1 . & 1 . & 50.70 & 9.1178 & 9.1178 \\ \text { LTE } & 1 . & 50.77 & 9.1178 & \end{array}$

2. $\mathrm{Ge}(\mathrm{Z}=32)$ at $\mathrm{kT}=500 \mathrm{eV} 0.01 \mathrm{~g} / \mathrm{cc} . \mathrm{L}_{\mathrm{R}}=46.87 \mathrm{~cm}$.

$\begin{array}{lllll}0.0000 & 0.11523 & 19.357 & 10.669 & 8.4899 \\ 0.0001 & 0.15234 & 21.924 & 10.546 & 8.8217 \\ 0.01 & 0.42188 & 29.126 & 10.265 & 10.007 \\ \text { LTE } & 0.5 & 29.745 & 10.244 & \\ \text { LASNEX } & & 23.377 & 10.482 & \end{array}$




\section{References.}

1. A. S. Edington. "The Internal Constitution of Stars". Cambridge University Press (1926).

2. R. P. Feynman, N. Metropolis and E. Teller, Phys. Rev. 75, 1561 (1949)

3. R. D. Cowan and J. Ashkin, Phys. Rev. 105, 144 (1957).

4. T. R. Carson, D. F. Mayers \& D. W. Stibbs. Mon. Not. R. Astr. Soc. 140, 483 (1968).

5. J. W. Zink, Phys. Rev. 176, 279 (1968)

6. B. F. Rozsnyai, Phys. Rev. A5, 1137 (1972).

7. B. H. Armstrong and R. W. Nicholls "Emission, Absorption and Transfer of Radiation in Heated Atmospheres". Pergamon (1972).

8. A. Goldberg, B. F. Rozsnyai and P. Thompson, Phys. Rev. A34, 421 (1986).

9. For a comprehensive report on hot plasma opacities see "WorkOp-III:94", edited by A. Rickert, $\mathrm{K}$. Eidmann, J. Meyer-Ter-Vehn, F. J. D. Serduke and C. A. Iglasias. Max-Planck Institut fur Quantenoptik, (1995).

10. D. R. Bates, F. R. S., A. E. Kingston and R. W. P. McWhirther, Proc.Roy. Soc. (London), A270, 297 (1962).

11. "A Time-Dependent Ionization Balance Model for Non-LTE Plasma" by Y. T. Lee, G. B. Zimmerman, D. S. Bailey, D. Dickson and D. Kim in "Radiative Properties of Hot Dense Matter III" edited by B. Rozsnyai, C. Hooper, R. Cauble, R. Lee and J. Davis. World Scientific, (1987).

12. D. Duston and J. Davis, Phys.Rev. A21, 1664 (1980).

13. D. Duston and J. Davis, Phys. Rev. A23, 2602 (1981).

14. J. P. Apruzese, J. Davis, D. Duston and K. G. Whitney, JQSRT 25, 479 (1981).

15. Y. T. Lee, JQSRT 38, 131 (1986).

16. B. F. Rozsnyai, JQSRT 17, 77 (1977).

17. W. Lotz, Astrophys. J. Suppl. XIV, 207, (1967).

18. B. F. Rozsnyai, V. L. Jacobs and J. Davis, Phys. Rev. A21, 1798 (1980). 
19. F. E. Irons, JQSRT 22, p1, 21, \& 37 (1979)

20. J. C. Weisheit, JQSRT 22, 585 (1979)

21. Y. T. Lee, R. A. London and G. B. Zimmerman, Phys. Fluids B 2, 2731 (1990).

22. M. Busquet, Phys. Fluids B5, 4191 (1993). 
Figure Captions.

Fig. 1. Photon confinement factors of praseodymium versus photon energy at $\mathrm{kT}=1 \mathrm{KeV}, 0.1 \mathrm{~g} / \mathrm{cc}$ density with $\mathrm{x}_{\mathrm{R}}=10^{-4}$ (I), $10^{-2}$ (II) and $\mathrm{x}_{\mathrm{R}}=1$ (III).

Fig. 2. Fermi functions of praseodymium at $\mathrm{kT}=1 \mathrm{KeV}$ and at $0.1 \mathrm{~g} / \mathrm{cc}$ density. The curves marked as (I), (II), (III) and (IV) correspond to $x_{R}=0,10^{-4}, 10^{-2}$ and 1 together with the LTE case, respectively.

Fig. 3. Comparison of the OLC and full non-LTE Fermi functions with the LTE case of praseodymium at $\mathrm{kT}=1 \mathrm{KeV}$ and at $0.1 \mathrm{~g} / \mathrm{cc}$ density with $\mathrm{x}_{\mathrm{R}}=0$.

Fig. 4 Same as Fig. 3 with $x_{R}=10^{-4}$

Fig. 5. Calculated opacities in $\mathrm{cm}^{-1}$ units of praseodymium at $\mathrm{kT}=1 \mathrm{KeV}$ and at $0.1 \mathrm{~g} / \mathrm{cc}$ density with $x_{R}=0$. Full curve - LTE, long dashed - full non-LTE based on the rate equations, short dashed LTE with the equivalent LTE temperature of $0.20313 \mathrm{KeV}$ from Table I.

Fig. 6. Same as Fig. 5 with $x_{R}=10^{-4}$.

Fig. 7. Same as Fig. 5 with $x_{R}=10^{-2}$

Fig. 8. Calculated opacities in $\mathrm{cm}^{-1}$ units of germanium at $\mathrm{kT}=0.5 \mathrm{KeV}$ and at $0.01 \mathrm{~g} / \mathrm{cc}$ density with $x_{R}=0$. Full curve - LTE, long dashed - full non-LTE based on the rate equations, short dashed LTE with the equivalent LTE temperature of $0.11523 \mathrm{KeV}$ from Table I.

Fig. 9. Same as Fig. 8 with $x_{R}=10^{-1}$.

Fig. 10. Same as Fig. 8 with $x_{R}=10^{-2}$

Fig. 11. Comparison of calculated opacities of germanium at $\mathrm{kT}=0.5 \mathrm{KeV}$ and at $0.01 \mathrm{~g} / \mathrm{cc}$ density with different $x_{R}-s$ with LASNEX prediction. Full curve $-x_{R}=10^{-4}$. long dashed $-x_{R}=10^{-2}$, short dashed - LASNEX.

Fig. 12. Fermi functions of praseodymium at $\mathrm{kT}=1 \mathrm{KeV}$ and at $0.1 \mathrm{~g} / \mathrm{cc}$ density with $\mathrm{x}_{\mathrm{R}}=10^{-4}$ with and without the Auger matrix elements in the rate equations.

Fig. 13. Opacitiy of praseodymium at $\mathrm{kT}=1 \mathrm{KeV}$ and at $0.1 \mathrm{~g} / \mathrm{cc}$ density with $\mathrm{x}_{\mathrm{R}}=10^{-4}$, with and without the Auger matrix elements in the rate equations, full and dashed curves, respectively. 


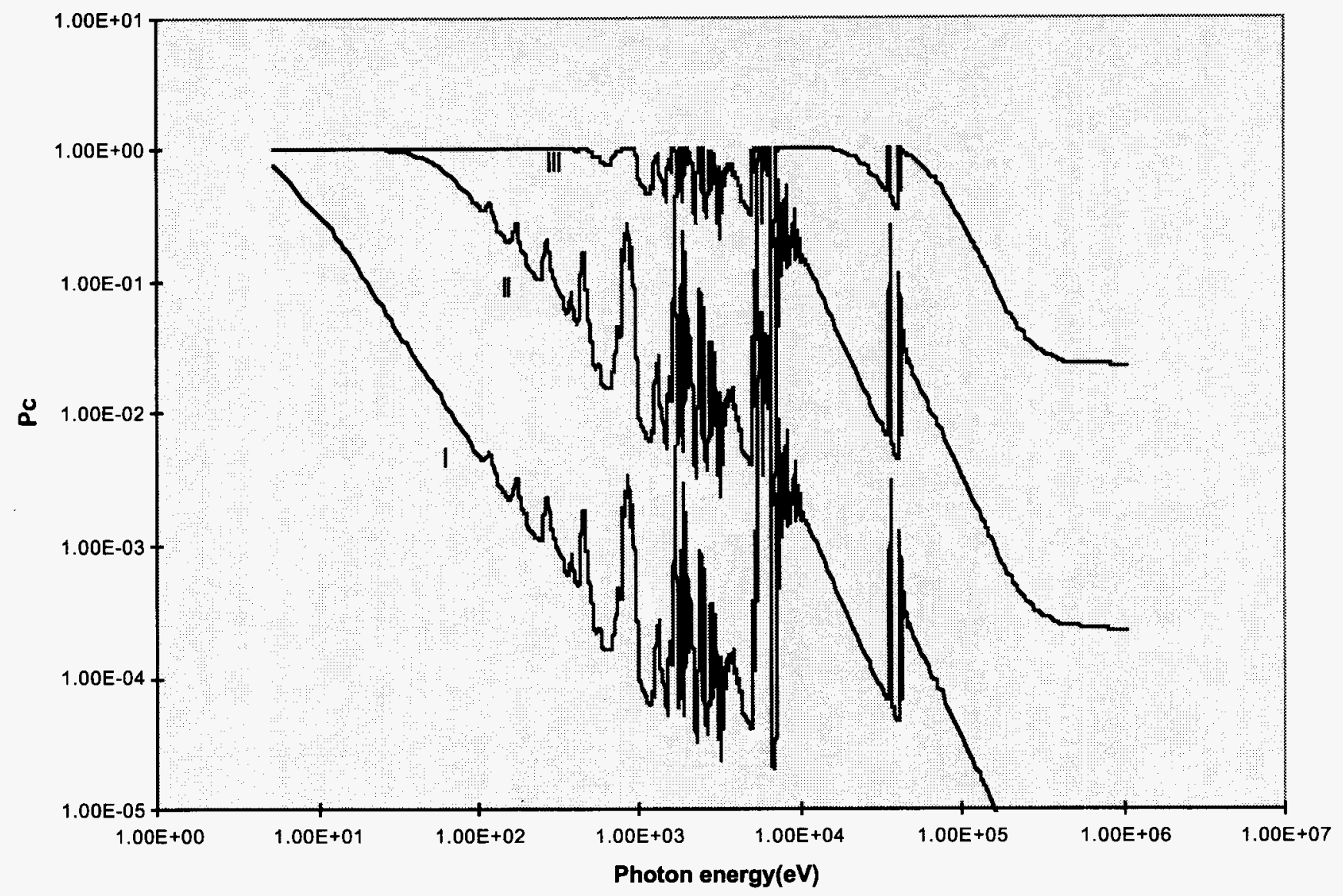

Fig. 1. Photon confinement factors of praseodymium versus photon energy at $\mathrm{kT}=1 \mathrm{KeV}, 0.1 \mathrm{~g} / \mathrm{cc}$ density with $\mathrm{x}_{\mathrm{R}}=10^{-4}(\mathrm{I}), 10^{-2}$ (II) and $\mathrm{x}_{\mathrm{R}}=1$ (III). 


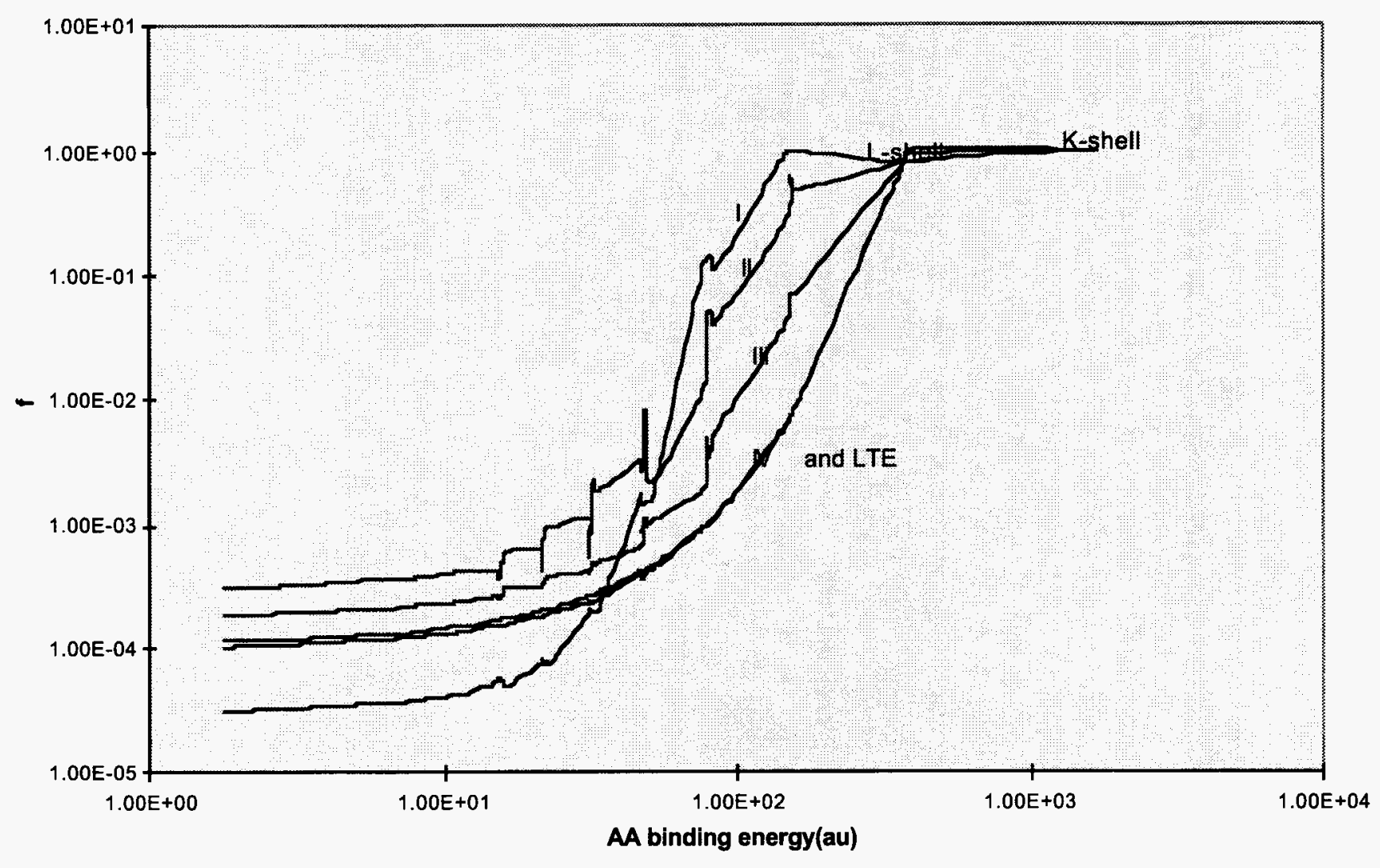

Fig. 2. Fermi functions of praseodymium at $\mathrm{kT}=1 \mathrm{KeV}$ and at $0.1 \mathrm{~g} / \mathrm{cc}$ density. The curves marked as (I), (II), (III) and (IV) correspond to $x_{R}=0,10^{-4}, 10^{-2}$ and 1 together with the LTE case, respectively. 


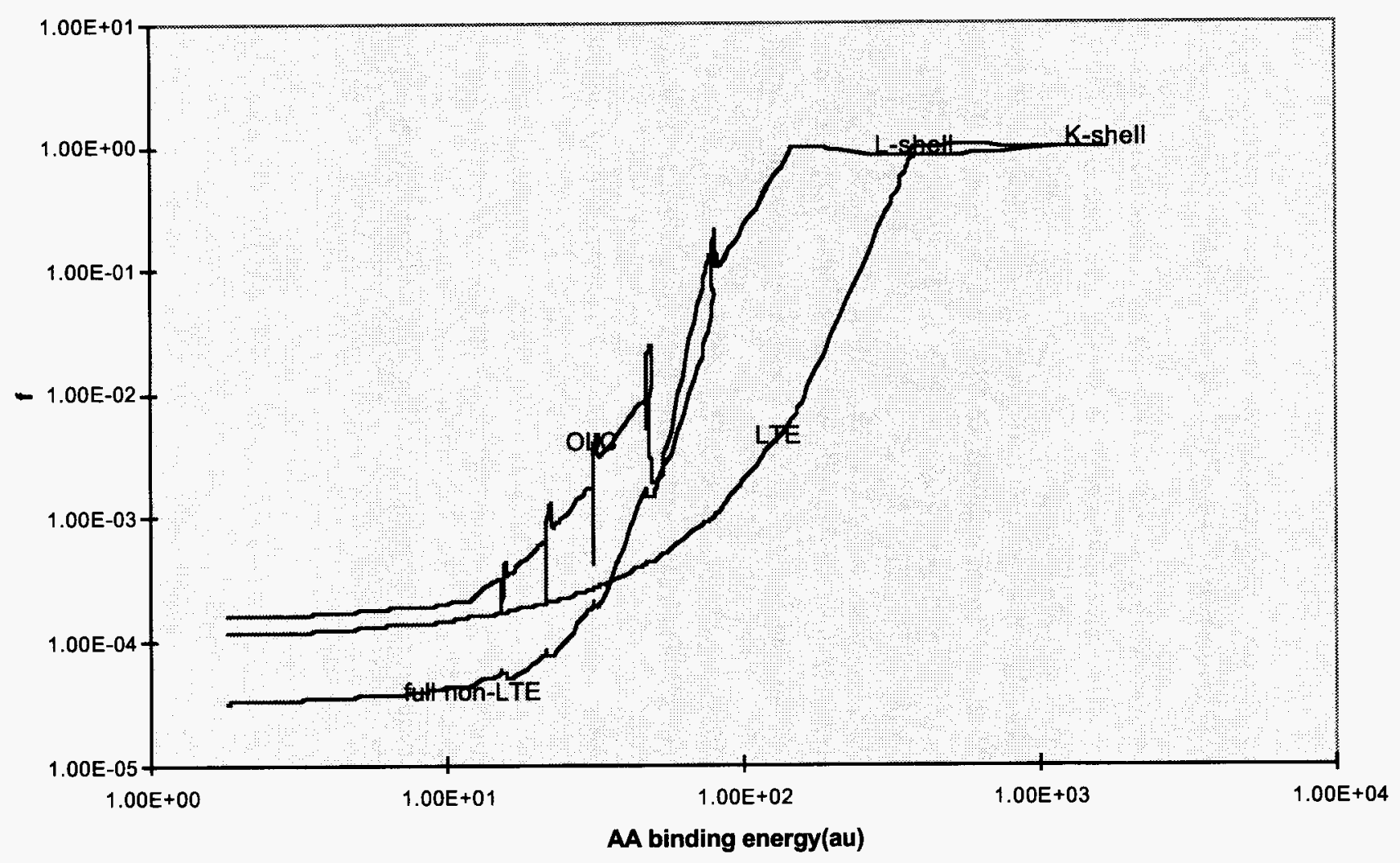

Fig. 3. Comparison of the OLC and full non-LTE Fermi functions with the LTE case of praseodymium at $\mathrm{kT}=1 \mathrm{KeV}$ and at $0.1 \mathrm{~g} / \mathrm{cc}$ density with $\mathrm{x}_{\mathrm{R}}=0$. 


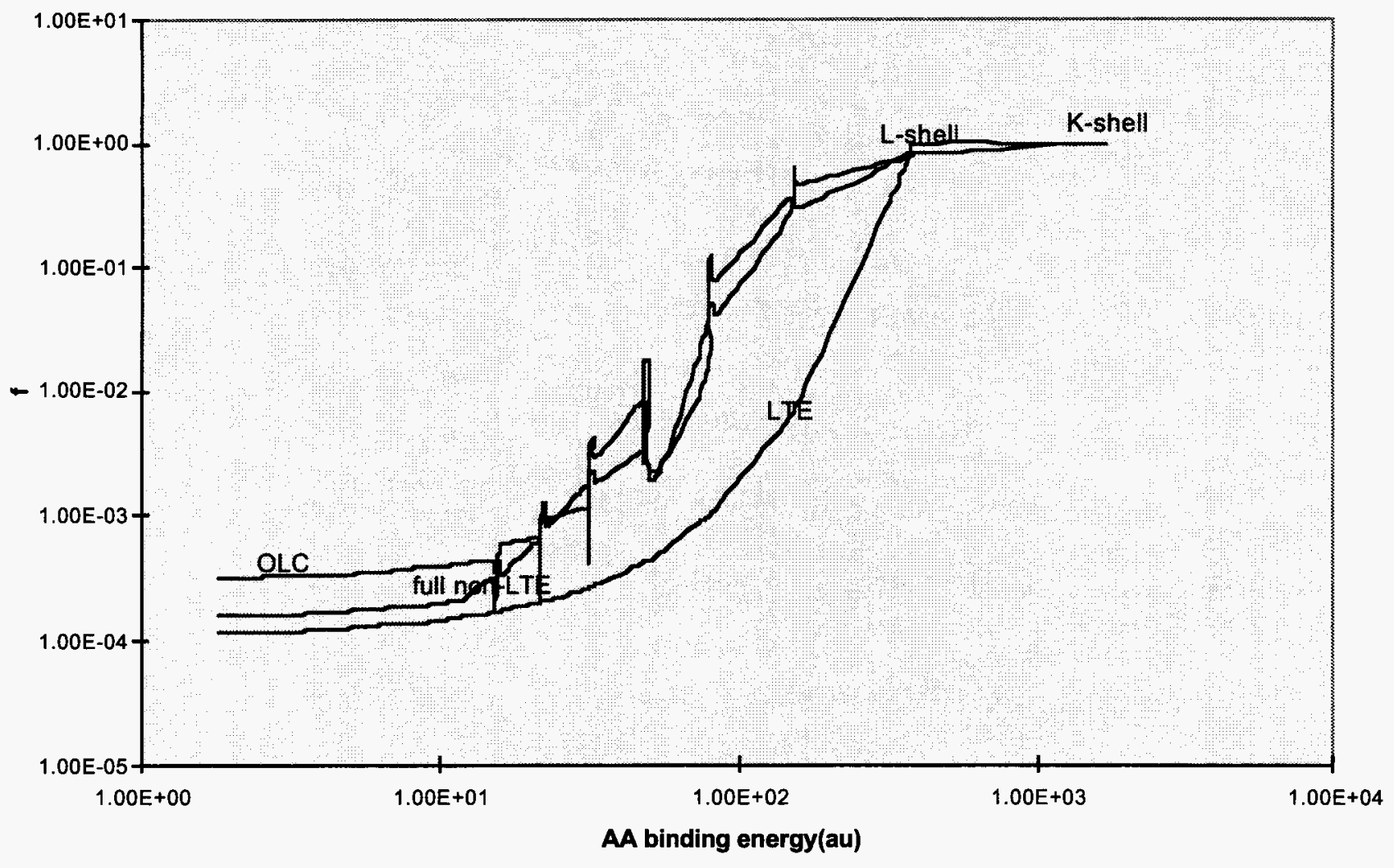

Fig. 4. Same as Fig. 3 with $x_{R}=10^{-4}$. 


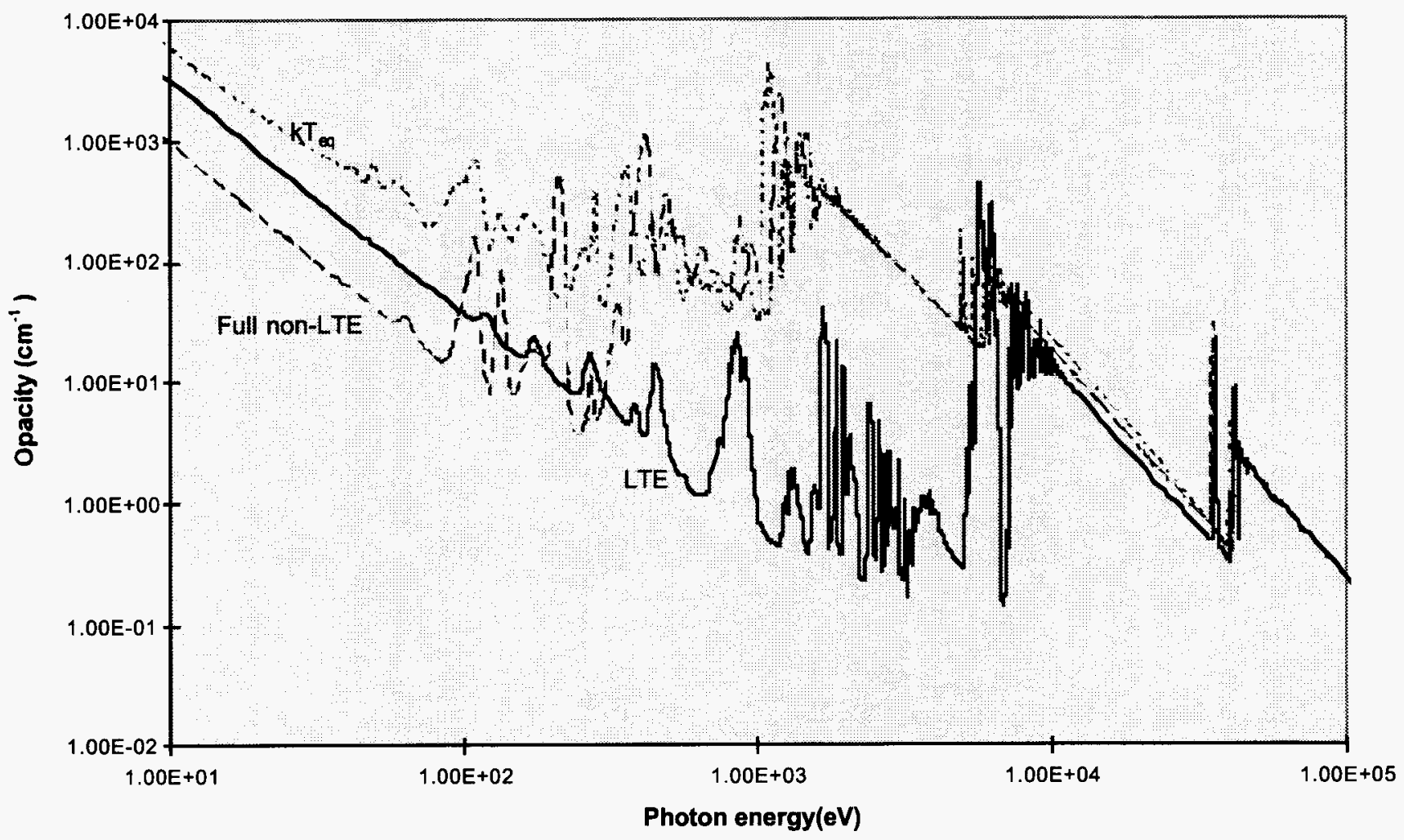

Fig. 5. Calculated opacities in $\mathrm{cm}^{-1}$ units of praseodymium at $\mathrm{kT}=1 \mathrm{KeV}$ and at $0.1 \mathrm{~g} / \mathrm{cc}$ density with $x_{R}=0$. Full curve - LTE, long dashed - full non-LTE based on the rate equations, short dashed LTE with the equivalent LTE temperature of $0.20313 \mathrm{KeV}$ from Table I. 


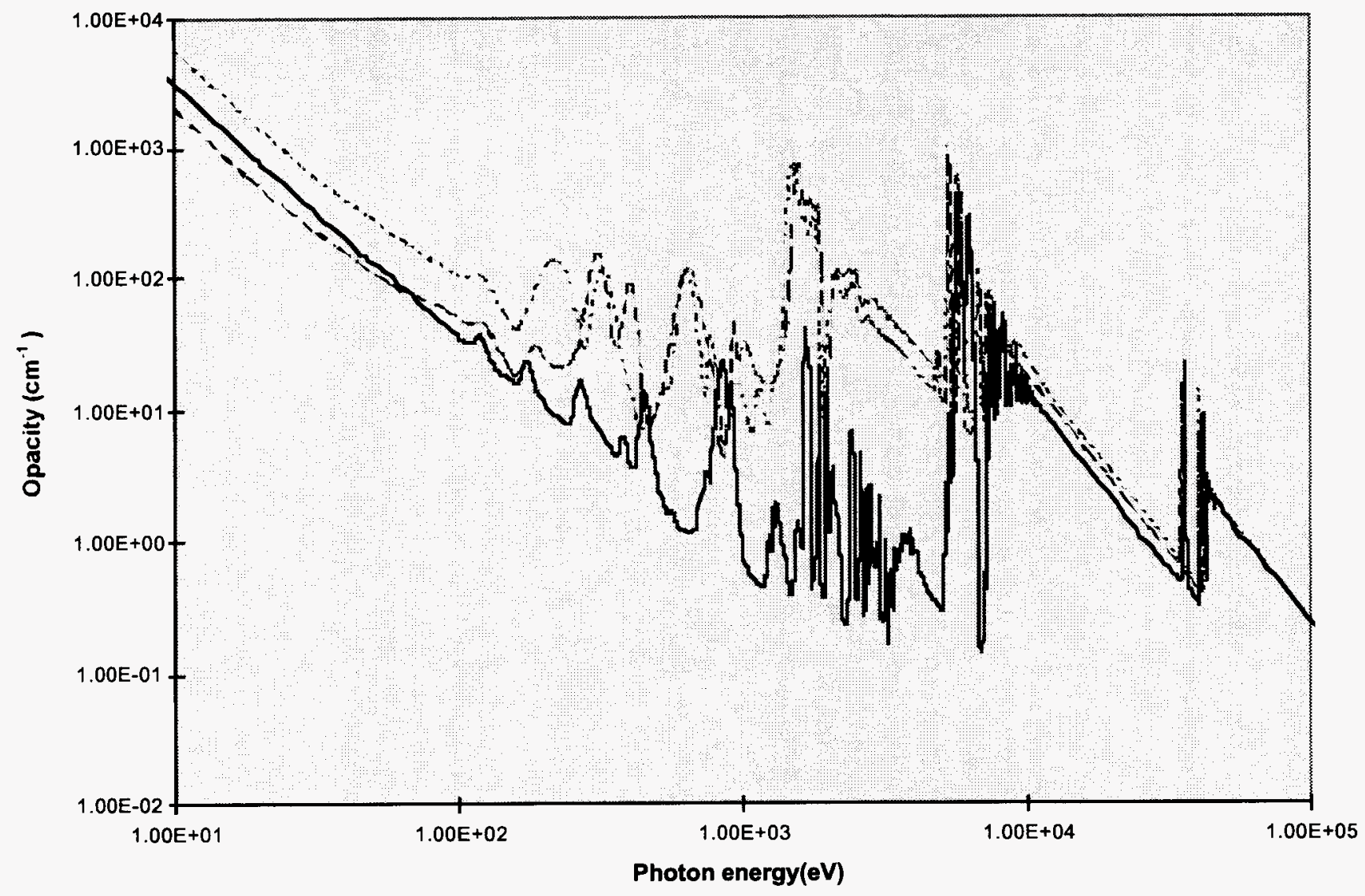

Fig. 6. Same as Fig. 5 with $x_{R}=10^{-4}$. 


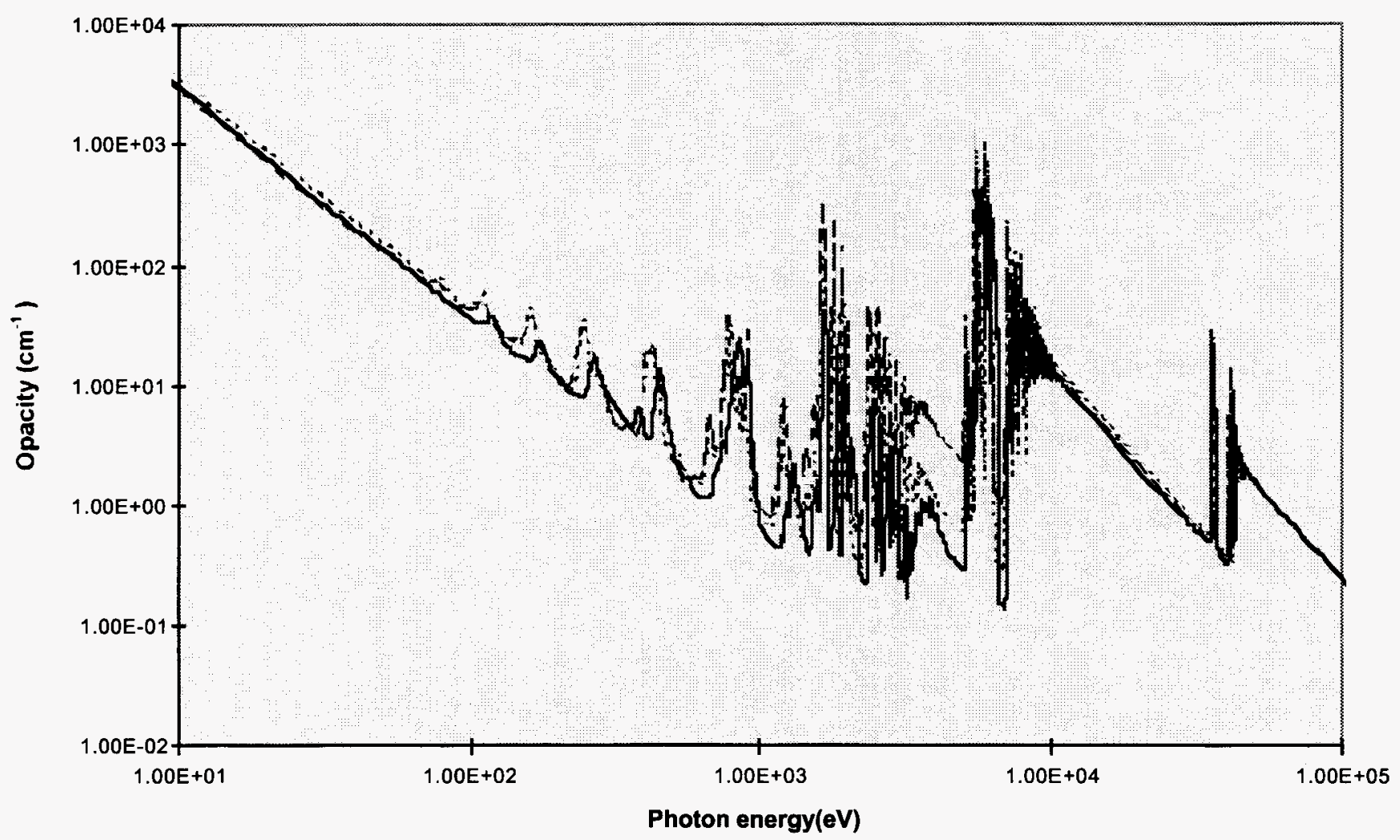

Fig. 7. Same as Fig. 5 with $x_{R}=10^{-2}$. 


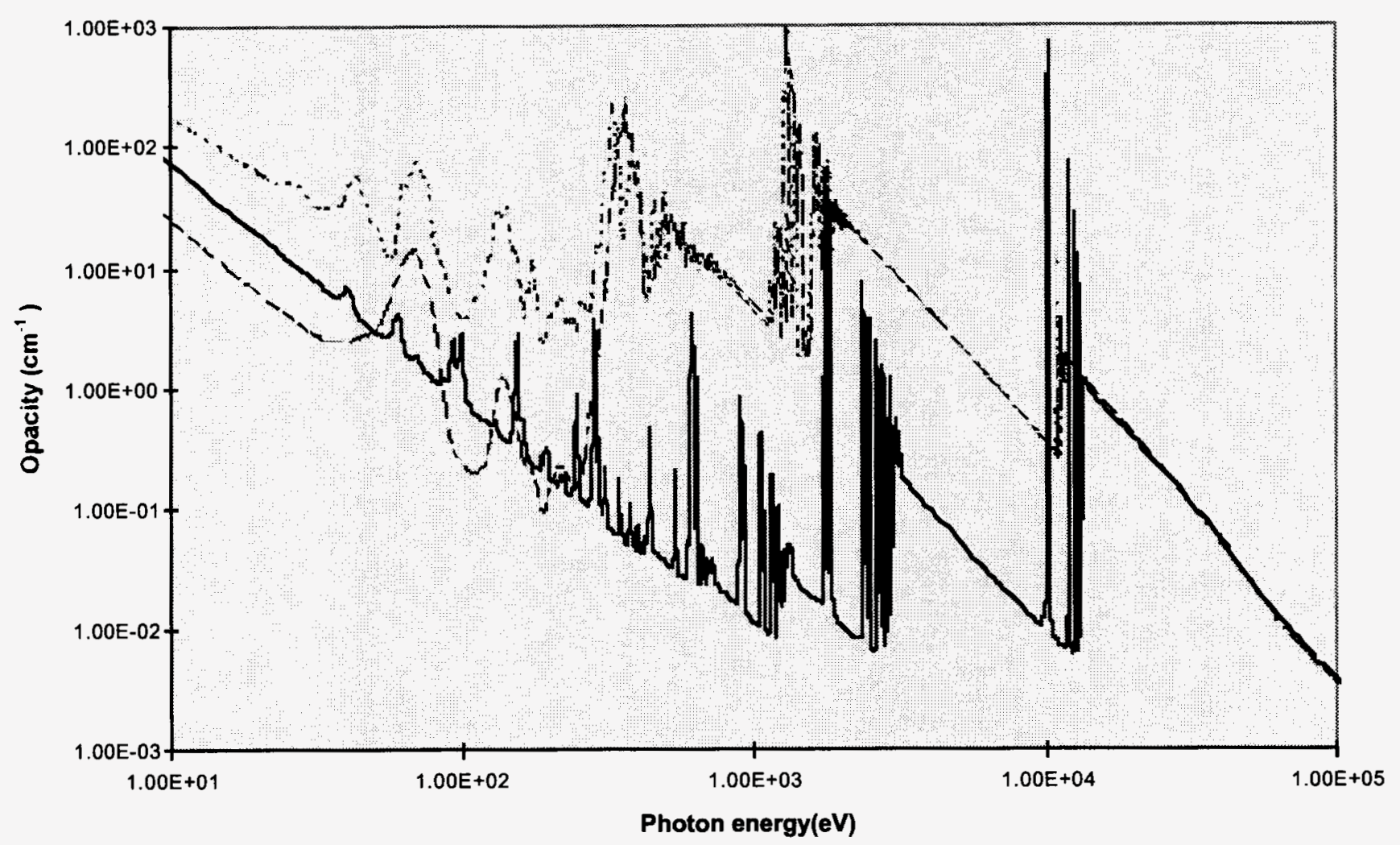

Fig. 8. Calculated opacities in $\mathrm{cm}^{-1}$ units of germanium at $\mathrm{kT}=0.5 \mathrm{KeV}$ and at $0.01 \mathrm{~g} / \mathrm{cc}$ density with $x_{R}=0$. Full curve - LTE, long dashed - full non-LTE based on the rate equations, short dashed LTE with the equivalent LTE temperature of $0.11523 \mathrm{KeV}$ from Table I. 


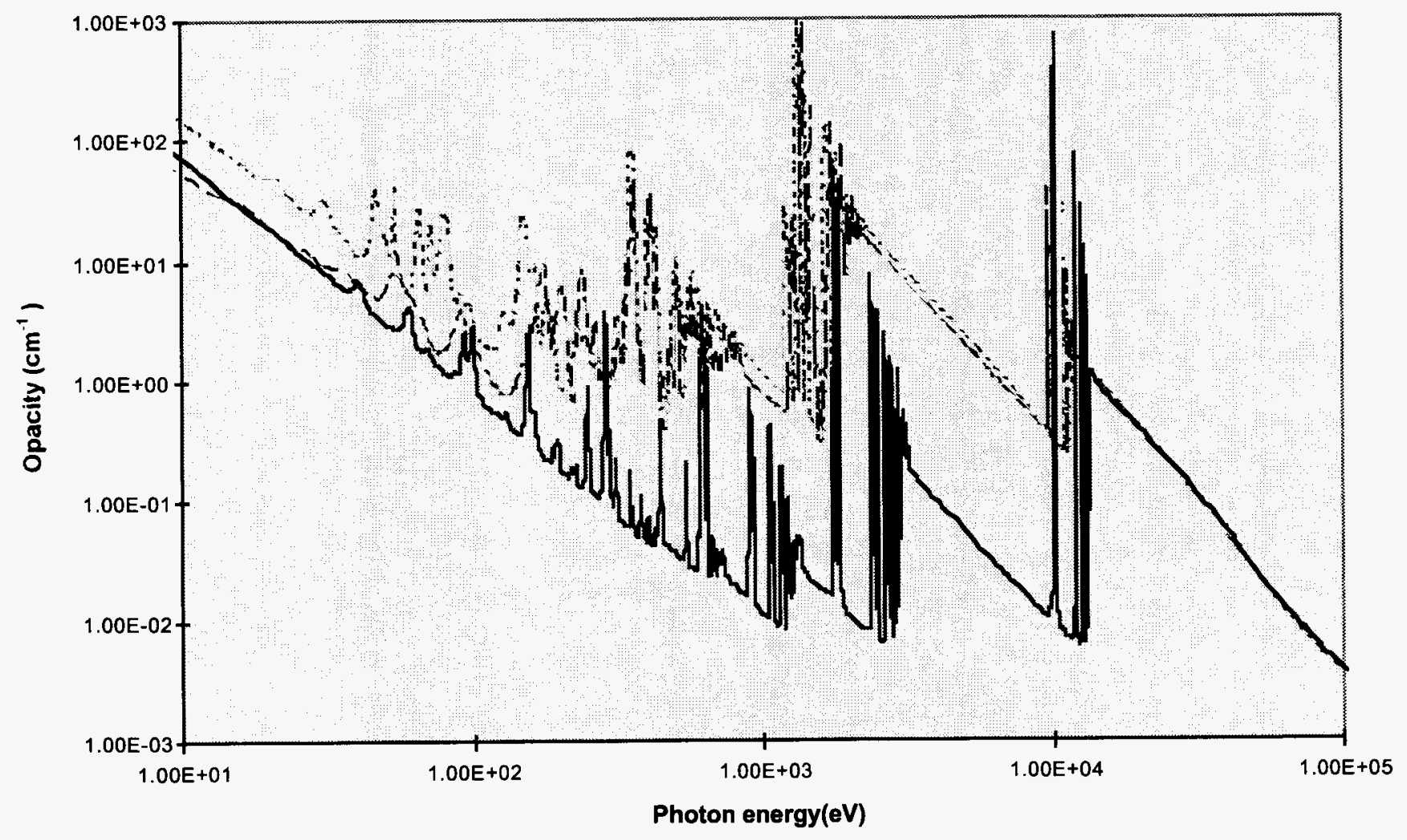

Fig. 9. Same as Fig. 8 with $x_{R}=10^{-4}$. 


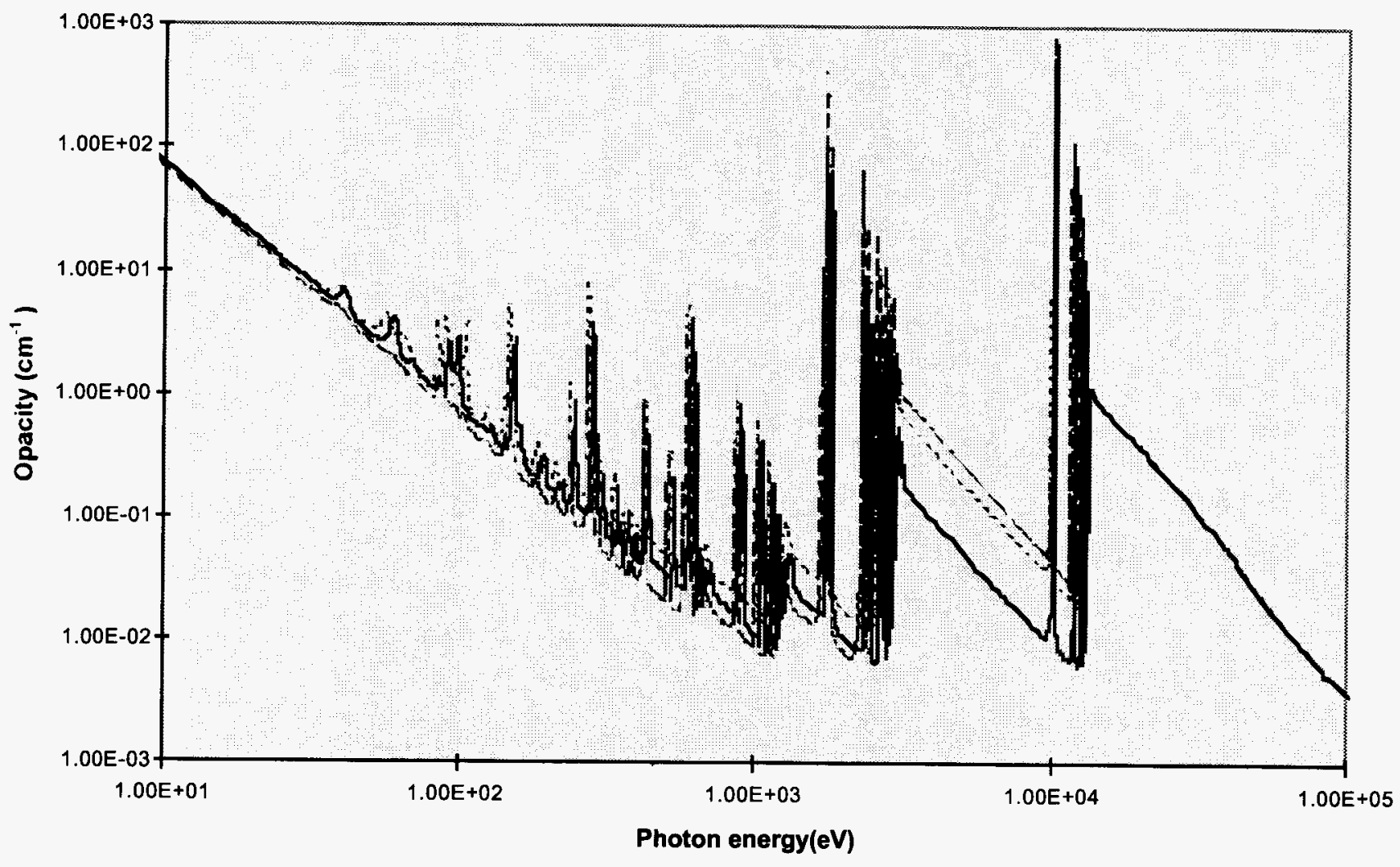

Fig. 10. Same as Fig. 8 with $x_{R}=10^{-2}$. 


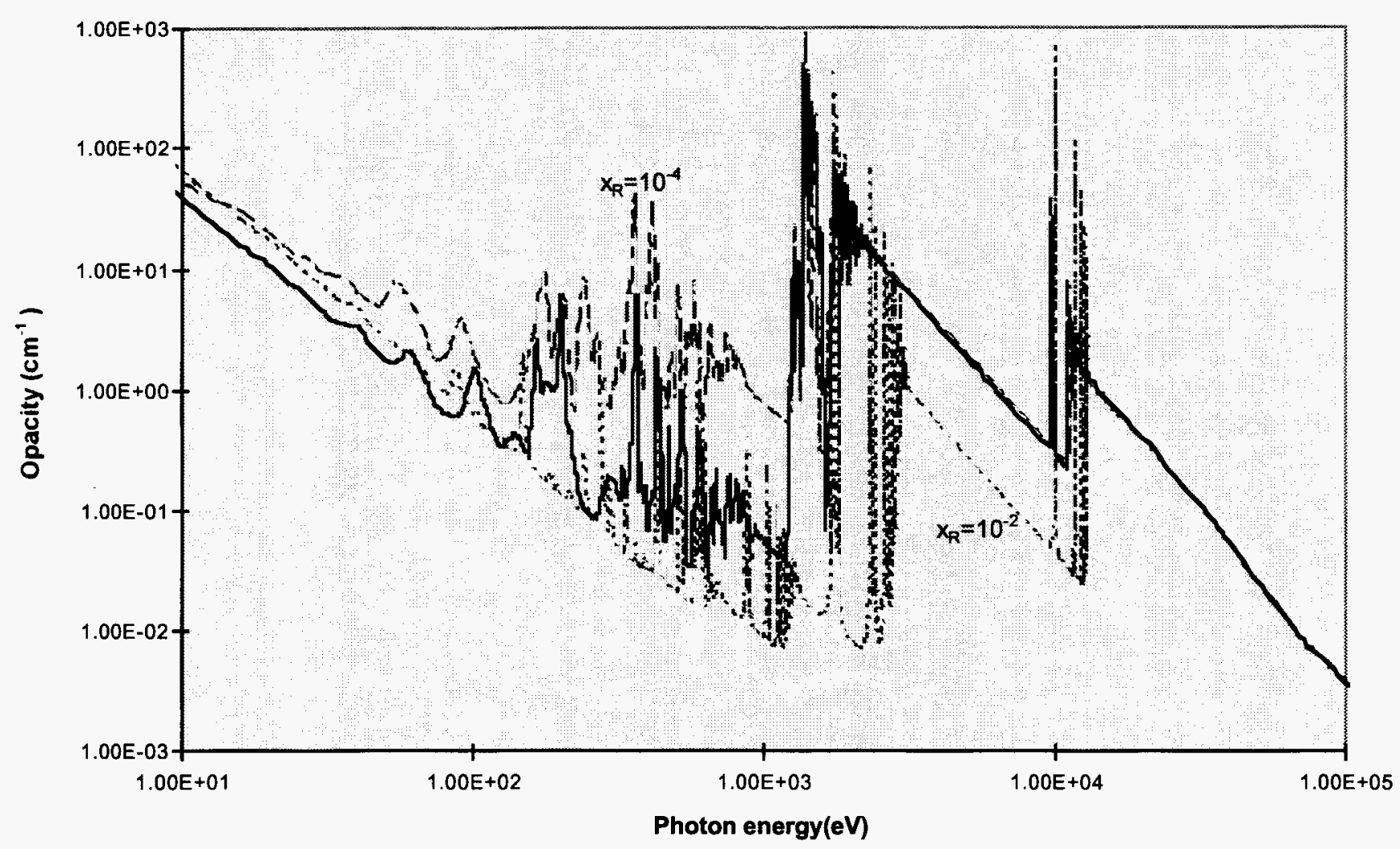

Fig. 11. Comparison of calculated opacities of germanium at $\mathrm{kT}=0.5 \mathrm{KeV}$ and at $0.01 \mathrm{~g} / \mathrm{cc}$ density with different $x_{R}-s$ with LASNEX prediction. Full curve - LASNEX, long dashed - $x_{R}=10^{-4}$, short dashed $-x_{R}=10^{-2}$. 


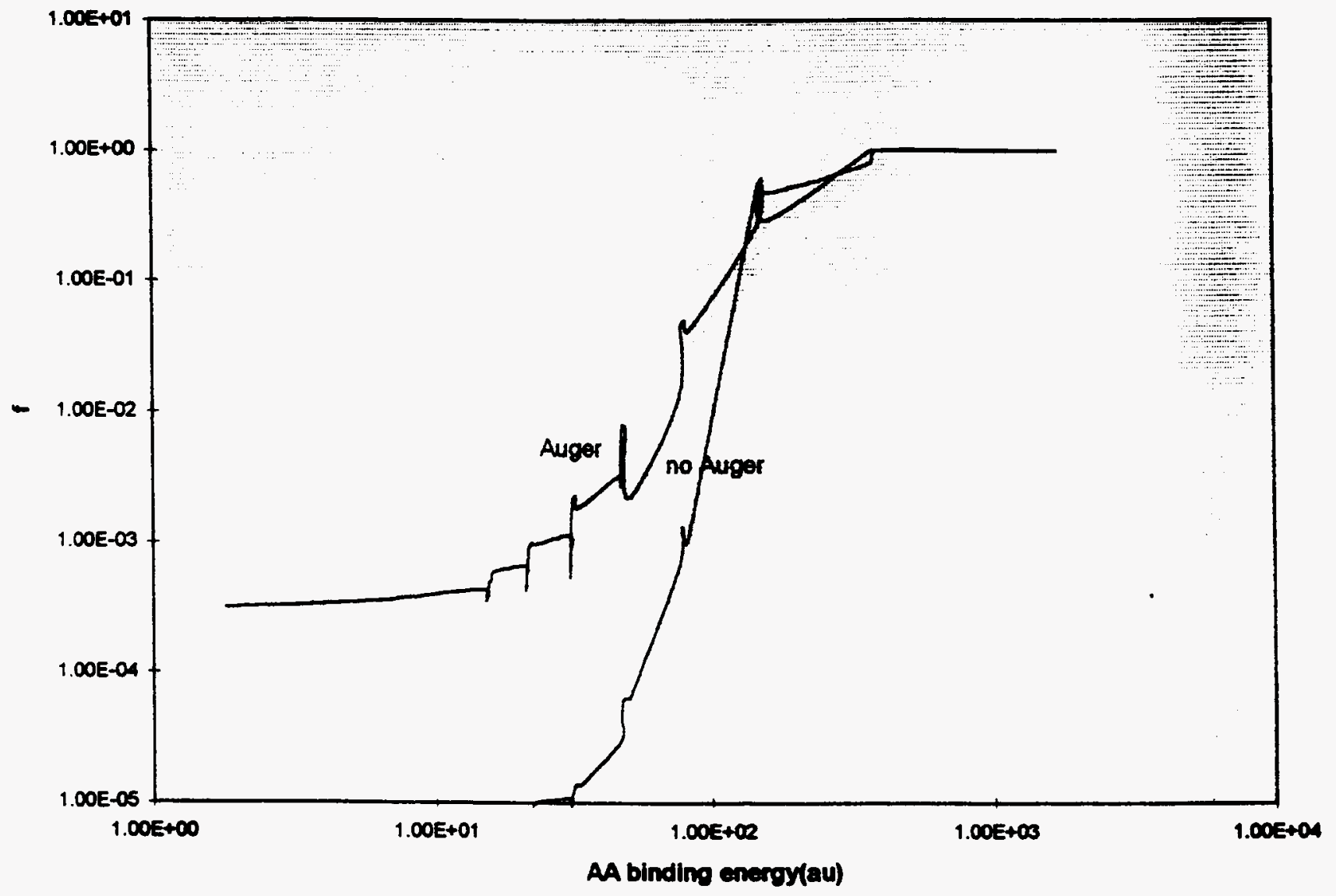

Fig. 12. Fermi functions of praseodymium at $k T=1 \mathrm{KeV}$ and at $0.1 \mathrm{~g} / \mathrm{cc}$ density with $\mathrm{x}_{\mathrm{R}}=10^{-4}$ with and without the Auger matrix elements in the rate equations. 


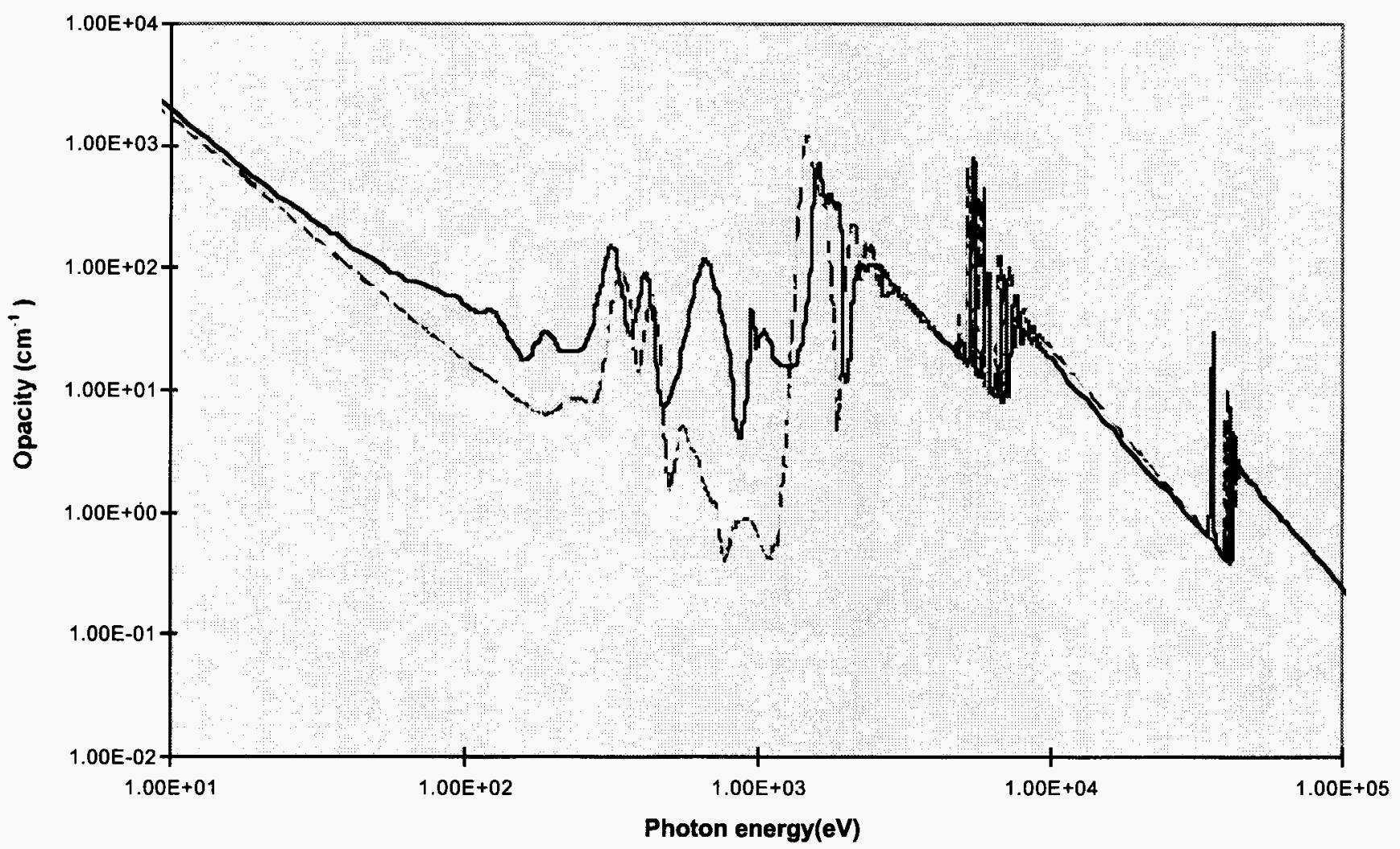

Fig. 13. Opacitiy of praseodymium at $\mathrm{kT}=1 \mathrm{KeV}$ and at $0.1 \mathrm{~g} / \mathrm{cc}$ density with $\mathrm{x}_{\mathrm{R}}=10^{-4}$, with and without the Auger matrix elements in the rate equations, full and dashed curves, respectively. 
Technical Information Department - Lawrence Livermore National Laboratory University of California • Livermore, California 94551

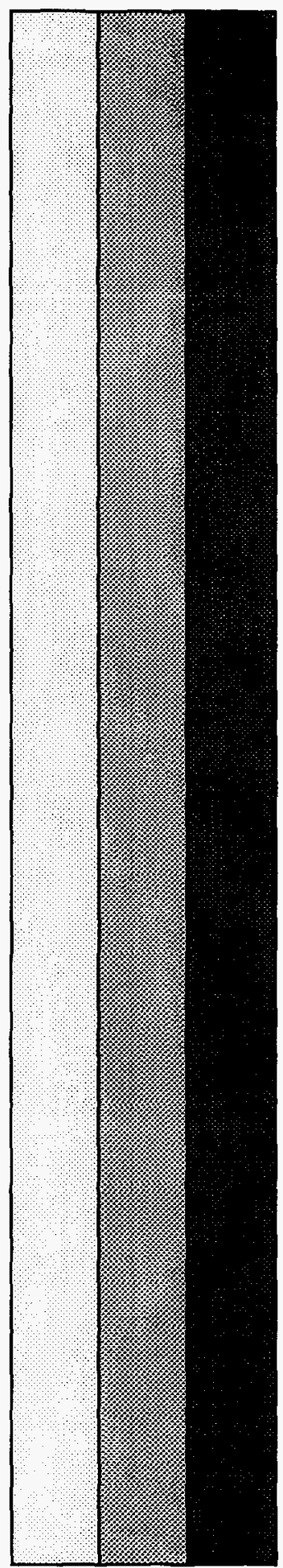

